\title{
Valores sociais sobre incorporação de tecnologias: o que pensam os participantes do SUS.
}

Social values on incorporation of technologies: what they think the participants of SUS.

Los valores sociales sobre la incorporación de tecnologías: lo que piensan los participantes del SUS.

\author{
Flávia Tavares Silva ELIAS ${ }^{1}$ \\ Kathiaja Miranda SOUZA ${ }^{2}$ \\ Augusto César MOTTA WILLER ${ }^{3}$ \\ Ana Paula C OLIVEIRA ${ }^{4}$ \\ Marcus Tolentino da SILVA ${ }^{5}$
}

RESUMO: $O$ trabalho teve como objetivo conhecer a opinião dos delegados da $14^{\text {a }}$ Conferência Nacional de Saúde, ocorrida no período de $1^{\circ}$ a 4 de dezembro de 2011, sobre os critérios de avaliação para incorporação de tecnologia para o sistema público de saúde no Brasil (SUS). Foi aplicado questionário para autopreenchimento aos visitantes do stand do Ministério da Saúde durante o evento. Um total de $11,5 \%$ dos participantes respondeu a pesquisa. Destes, $43,6 \%$ representaram cidadãos ou pacientes; $33,6 \%$ dos profissionais de saúde, $13,1 \%$ tomadores de decisão; $3,8 \%$ prestadores de serviços e 5,9\% outros. A maioria já havia participado do controle social do SUS, há mais de cinco anos. Os critérios pré-definidos para a avaliação das tecnologias no questionário foram colocados em uma ordem hierárquica: a melhoria da qualidade de vida e sobrevida dos pacientes (60\%); evidências sobre os efeitos previstos na prevenção, diagnóstico, tratamento ou reabilitação (49\%); evidências sobre a segurança para o paciente / usuário / consumidor (46\%); impacto sobre a saúde da população (43\%); relação entre benefícios / efeitos da tecnologia e os custos (40\%). Os «custos para o sistema de saúde» e «critérios de custos para os doentes» estavam em penúltimo e último lugar, respectivamente. As perspectivas dos doentes / cidadãos e profissionais de saúde devem ser estimulados a fim de apoiar a aplicação da Lei 12.401 / 2011, que estabelece critérios para Incorporação de Tecnologias da Saúde.

Palavras-chave: valores sociais, conferências de saúde, Avaliação da Tecnologia Biomédica

\footnotetext{
${ }^{1}$ Pesquisador em saúde pública na Fiocruz Brasília, perfil avaliação de tecnologia e de programas em saúde. Brasília, DF. E-mail: flavia.tselias@gmail.com

${ }^{2}$ Mestrado em Saúde Coletiva pela Universidade de Brasília, Brasil(2014) Consultoria Técnica do Ministério da Saúde , Brasil.

3 Especialização em Gestão Estratégica de Organizações Públicas pela Faculdade Projeção, Brasil (2011) Analista de Ciência e Tecnologia do Conselho Nacional de Desenvolvimento Científico e Tecnológico , Brasil.

${ }^{4}$ Universidade Nova de Lisboa, Lisboa, Portugal.

${ }^{5}$ Universidade Federal do Amazonas.
} 
ABSTRACT : the survey had the objective to become aware of the opinion of the delegates from the 14th National Health Conference during the period of the 1st to the 4th of December, 2011. About the technology assessment criteria for the public health system in Brazil (SUS). A self-questionnaire survey was applied to all visitors at a Ministry of Health booth in the event. About 11.5\% (502/4370) participants were covered by the survey. Of these, $43.6 \%$ represented citizens/patients; $33.6 \%$ health professionals, $13.1 \%$ policymakers; $3.8 \%$ service providers and5.9\% others. The majority of them have participated in SUS social control for more than five years. Eleven of the pre-defined criteria for technology assessment on the questionnaire were put in a hierarchical order in the following manner: improved quality of life and patient survival $(60 \%)$; evidence on the anticipated effects in prevention, diagnostics, treatment or rehabilitation (49\%); evidence regarding safety for patient/user/consumer (46\%); impact on the population's health (43\%); relationship between benefits/effects of the technology and the costs (40\%). The criteria "costs for the health system" and "costs for patients" were in second to last and last place, respectively. The perspectives of patients/citizens and health professionals should be stimulated in order to support the application of the Law 12,401/2011, which establishes criteria for Incorporation of Health Technologies.

Keywords: social values, health conferences, Technology Assessment, Biomedical

RESUMEN: la investigación tuvo el objectivo de conocer la opinión de los delegados de la $14^{\text {a }}$ Conferencia Nacional de Salud, durante la duración del 1 al 04 diciembre de 2011,.en los criterios de evaluación para la incorporación de la tecnología en el sistema de salud pública en Brasil (SUS). Fue submetido a los visitantes del stand del Ministério de la Salud un auto-cuestionario. Al menos 11,5\% (502/4370) de los participantes estaban cubiertos por la encuesta. De estos, el 43,6\% representado ciudadanos o pacientes; $33,6 \%$ de los profesionales de la salud, 13,1\% de los que toman las decisiones; $3,8 \%$ los proveedores de servicios y $5,9 \%$ otros. La mayoría de ellos han participado en el control social del SUS por más de cinco años. Once de los criterios predefinidos para la evaluación de las tecnologías en el cuestionario fueron colocados en un orden jerárquico de la siguiente manera: la mejora de la calidad de vida y la supervivencia de los pacientes (60\%); pruebas para los fines establecidos en la prevención, diagnóstico, tratamiento o rehabilitación (49\%); evidencia sobre la seguridad para el paciente / usuario / consumidor (46\%); impacto en la salud de la población (43\%); beneficios de relación / efectos de la tecnología y el costo (40\%).

El «costo para el sistema de salud»y «criterios de costo para los pacientes» fueron penúltimo y último, respectivamente. La perspectiva de los pacientes / ciudadanos y profesionales de la salud deben ser alentados a apoyar la aplicación de la Ley 12.401 / 2011 que establecen criterios para la Incorporación de tecnologias sanitarias

Palabras llaves: valores sociales, conferencias de Salud, Evaluación de la Tecnología Sanitaria. 


\section{INTRODUÇÃO}

A obtenção de melhor qualidade e sustentabilidade nos sistemas de saúde de caráter universalreside no fato de que tecnologias disponíveis nos serviços têm implicação direta na manutenção da saúde e na qualidade de vida do usuário (1), (2). Por isso a importância da Avaliação de Tecnologia em Saúde (ATS), direcionar os processos de cobertura e financiamento de medicamentos, procedimentos, equipamentos e testes diagnósticos (1).

Por essa importância, a participação de usuários e profissionais $(4,5)$, na definição de prioridades e processo de ATS (6) tornou-se essencial para a garantia da qualidade nos cuidados à saúde (3). Porém, permanece aberto o debate sobre as formas de participação de pacientes, usuários e consumidores na definição dos critérios requeridos para a ATS, embora as experiências internacionais reafirmam a importância do envolvimento desse público nos processos de avaliação e incorporação de tecnologias (6), (7).

A sociedade Health Tecnology Assessment International, do Reino Unido (8), reforça que a visão e os valores subjetivos dos pacientes devem ser colocados em destaque na priorização e na alocação de recursos quando se tratar de novas tecnologias para a saúde. E essa participação ativa da sociedade tem sido objeto de debate em diversos fóruns internacionais (5),(7).

No Brasil, as atividades de ATS iniciaram em 2004, no Ministério da Saúde, com a criação de uma política de pesquisa estratégica (9). Em 2006, ganhou maior foco com a formalização de estratégias para análise de incorporação e exclusão de tecnologias financiadas pelo Sistema Único de Saúde (SUS) (10), dentre elas a criação de uma rede que envolve serviços, academia e gestão, a Rede Brasileira de Avaliação de Tecnologias em Saúde (REBRATS).

A REBRATS está em expansão e o seu potencial ainda é inexplorado pela sociedade que passa a ser requerida, cada vez mais, para uma participação ativa nos debates sobre os processos de incorporação de tecnologias no Sistema Único de Saúde (2), (11).

$\mathrm{Na}$ adoção e uso de tecnologias em saúde devem ser observados critérios éticos, políticos, sociais e econômicos. Mas a sociedade compreenderia tais critérios como suficientes para a adoção e uso de tecnologias em saúde?

Para responder a essa pergunta, fez-se um inquérito junto ao público da $14^{\mathrm{a}}$ Conferência Nacional de Saúde, realizada entre os dias 30 de novembro a 04 de dezembro de 2011, em Brasília/DF. O intuito foi conhecer quais os critérios devem ser adotados para o uso de tecnologias em saúde. A pesquisa também indagou sobre o grau de conhecimento sobre a Rede Brasileira de Avaliação de Tecnologias em Saúde (REBRATS) junto àquele público. 


\section{METODOLOGIA}

Desenvolveu-se um inquérito institucional, conduzido pelo Ministério da Saúde, durante a $14^{\text {a }}$ Conferência Nacional de Saúde, em Brasília/DF (12).

Os participantes da conferência preencheram um questionário contendo onze perguntas, com questões estruturadas. Dois documentos foram considerados para a construção das perguntas e opções de respostas. Um sobre o envolvimento de pacientes nas avaliações de tecnologias (7) e outro sobre critérios de priorização para definição de estudos a serem apoiados pelo Ministério da Saúde (10).

As perguntas fechadas com opções de respostas foram: $\left(1^{\mathrm{a}}\right)$ Qual segmento você representa na $14^{\mathrm{a}}$ Conferência Nacional de Saúde? ( $2^{\mathrm{a}}$ ) Escolaridade ( $3^{\mathrm{a}}$ ) Tempo envolvido no controle social do Sistema Único de Saúde (4 $4^{a}$ Tipos de tecnologias em saúde (5) Meios que utiliza para obter informações sobre tecnologias em saúde $\left(6^{a}\right)$ Principais aspectos envolvidos no uso de tecnologias em saúde $\left(7^{\mathrm{a}}\right)$ Critérios que utilizaria para avaliar tecnologias em saúde: Melhoria da qualidade de vida e sobrevida do paciente, Evidências dos efeitos esperados na prevenção, diagnóstico, tratamento ou reabilitação, Evidências dos efeitos esperados na prevenção, diagnóstico, tratamento ou reabilitação, Impacto sobre a saúde da população, Relação entre benefícios/efeitos das tecnologias e os custos, Evidências de benefícios em relação às tecnologias existentes, Implicações para os serviços de saúde, Custos para o sistema de saúde, Custos para pacientes. ( $8^{\mathrm{a}}$ ) Conhecimento sobre o que é Avaliação de Tecnologias em Saúde $\left(9^{a}\right)$. Reconhecimento de instituições/documentos envolvidos com Avaliação de Tecnologias em Saúde.

Perguntas abertas: $\left(10^{\mathrm{a}}\right)$ Gostaria de receber informes, materiais, resultados de pesquisas técnicocientíficas sobre Avaliação de Tecnologias em Saúde relevantes para a gestão do Sistema Único de Saúde? (11 $1^{\mathrm{a}}$ ) Gostaria de participar do processo de Avaliação de Tecnologias em Saúde?

Para caracterização da população alvo, procedeu-se proporção simples das variáveis: segmento, escolaridade e tempo envolvido no controle social. Usou-se o mesmo procedimento para ranquear os critérios para avaliar as tecnologias em saúde, para identificar as fontes de informação e as instituições e documentos relacionados com avaliação de tecnologias. Quando possível, os resultados foram estratificados pelo segmento e pelo tempo envolvido no controle social. As diferenças entre os estratos foram averiguadas pelo teste do chi-quadrado.

Para investigação dos fatores associados ao critério mais selecionado, custo e conhecimento da REBRATS, criou-se um modelo de regressão logística com variância robusta do tipo stepwise. Nesse modelo, consideraram-se como variáveis dependentes as opções da primeira, segunda, terceira, quinta, sétima, oitava, nona e décima pergunta. A partir de todas as variáveis analisadas, foram excluídas aquelas sem significância estatística ( $\mathrm{p}>0,05)$. Calculou-se a odds ratio $(\mathrm{OR})$ dos fatores associados identificados. O modelo foi repetido para segmentos sociais mais representativos. Todos os cálculos foram executados no pacote estatístico STATA, versão 10. 


\section{RESULTADOS}

A $14^{\text {a }}$ Conferência Nacional de Saúde teve a participação de 3.428 pessoas, das quais 502 $(14,6 \%)$ participaram do inquérito. As características dos participantes estão descritas na Tabela 1. A maior parte dos entrevistados foi composta pelo segmento de usuários, por pessoas com nível superior e com mais de oito anos de atividade no controle social do Sistema Único de Saúde.

Segundo normativa do Conselho Nacional de Saúde ${ }^{12}$, metade dos participantes da $14^{\text {a }}$ Conferência Nacional de Saúde teve sua composição formada pelo segmento dos usuários do sistema de saúde.

Tabela 1. Características da população em estudo, inquérito na $14^{\mathrm{a}} \mathrm{CNS},(\mathrm{n}=502)$

$\begin{array}{ll}\text { Característica } & \% \\ \text { Segmento social } & \\ \text { Usuário/paciente/consumidor } & 43,8 \\ \text { Profissional de saúde } & 33,8 \\ \text { Gestor } & 13,2 \\ \text { Prestador } & 3,8 \\ \text { Outros } & 5,4 \\ \text { Escolaridade } & \% \\ \text { Superior } & 68,1 \\ \text { Médio } & 28,1 \\ \text { Fundamental } & 3,8 \\ \text { Tempo envolvido no controle social } & \% \\ 0 \text { a } 4 \text { anos } & 29,5 \\ 4 \text { a } 8 \text { anos } & 21,9 \\ 8 \text { a } 15 \text { anos } & 25,7 \\ >15 \text { anos } & 22,9\end{array}$

Fonte: questionários respondidos

O critério mais frequente para avaliar tecnologias em saúde, escolhido por $60,4 \%$ dos sujeitos respondentes, foi a melhoria da qualidade de vida e sobrevida do paciente. Dentre os respondentes, 36,3\% marcaram ao menos uma opção relacionada ao custo como critérios para avaliar tecnologias em saúde. (Tabela 2). Quando estratificados pelo segmento social, os prestadores de serviços ao SUS preferiram o critério impacto sobre a saúde da população como o critério mais relevante. Não houve diferença de hierarquia entre os demais segmentos.

Tabela 2. Critérios de avaliação de tecnologias em saúde selecionados pelos participantes, inquérito na $14^{\mathrm{a}} \mathrm{CNS},(\mathrm{n}=502)$

\begin{tabular}{|c|c|}
\hline Critérios de valores sociais & $\%$ \\
\hline Melhoria da qualidade de vida e sobrevida do paciente & 60,4 \\
\hline $\begin{array}{c}\text { Evidências dos efeitos esperados na prevenção, diagnóstico, } \\
\text { tratamento ou reabilitação }\end{array}$ & 48,8 \\
\hline Evidências de segurança para o paciente/usuário/consumidor & 46,4 \\
\hline
\end{tabular}




\begin{tabular}{|c|c|}
\hline Impacto sobre a saúde da população & 42,6 \\
\hline Relação entre benefícios/efeitos das tecnologias e os custos & 40,2 \\
\hline Evidências de benefícios em relação às tecnologias existentes & 34,1 \\
\hline $\begin{array}{c}\text { Implicações para os serviços de saúde (praticidade de uso, equidade } \\
\text { no acesso) }\end{array}$ & 33,5 \\
\hline Frequência e gravidade da doença & 33,5 \\
\hline Expectativas e conhecimentos dos usuários & 27,5 \\
\hline Custos para o sistema de saúde & 25,5 \\
\hline Custos para pacientes & 23,9 \\
\hline
\end{tabular}

Fonte: questionários respondidos

Os fatores associados ao critério "melhoria da qualidade de vida e sobrevida do paciente" foram avaliados por análises multivariadas que compararam os participantes que optaram por esse critério com aqueles que não o fizeram. A tabela 3 mostra fatores estatisticamente significativos das análises multivariadas. Os achados revelaram: a) quando todos os participantes são considerados, há maior preferência em receber informações sobre avaliação de tecnologias em saúde, em detrimento de participar do processo. b) Provavelmente, as respostas dos usuários tiveram maior influência nos resultados na população estudada. c) Observou-se que dois critérios, "efeitos observados na prevenção, diagnóstico, tratamento ou reabilitação" e "implicações para os serviços de saúde", foram estatisticamente mais selecionados, quando estratificados pelos profissionais de saúde.

Tabela 3. Entendimento dos participantes quanto à existência da Rede Brasileira de Avaliação de Tecnologias em Saúde, inquérito na $14^{\mathrm{a}} \mathrm{CNS},(\mathrm{n}=502)$

\begin{tabular}{|c|c|c|c|}
\hline Participantes & $\%$ & IC95\% & $\mathrm{p}$-valor \\
\hline Todos & 27,3 & $23,5-31,3$ & \\
\hline Segmento social & & & 0,768 \\
\hline Usuário/paciente/consumidor & 25,1 & $19,7-31,2$ & \\
\hline Gestor & 33,3 & $22,8-45,3$ & \\
\hline Prestador & 26,3 & $10,3-49,1$ & \\
\hline Profissional de saúde & 27,2 & $20,9-34,3$ & \\
\hline Outros & 29,6 & $14,8-48,6$ & \\
\hline Tempo envolvido no controle social & & & 0,996 \\
\hline 0 a 4 anos & 27,5 & $20,6-35,4$ & \\
\hline 4 a 8 anos & 26,4 & $18,8-35,2$ & \\
\hline 8 a 15 anos & 26,4 & $19,3-34,5$ & \\
\hline$>15$ anos & 27,0 & $19,5-35,6$ & \\
\hline
\end{tabular}

Fonte: questionários respondidos

A análise multivariada apontou que o segmento de usuários do SUS tem maior chance de priorizar a frequência e gravidade da doença $(\mathrm{p}<0,001)$ e o impacto sobre a saúde da população ( $<<0,001)$, de conhecerem o Boletim Brasileiro de Avaliação de Tecnologias em Saúde (BRATS) $(\mathrm{p}=0,008)$ e, de não querer participar do processo de avaliação de tecnologias em saúde ( $\mathrm{p}=0,017)$. 
A internet foi assinalada como fonte de informação por $82,3 \%$ dos participantes, que elencaram o critério melhoria da qualidade de vida e sobrevia do paciente (Tabela 3). As revistas especializadas da área da saúde foram apontadas por 56,4\% dos conferencistas, e 48,8\% desses marcaram a participação em cursos, com destaque ao segmento de prestadores $(73,7 \% ; \mathrm{p}=0,005)$.

Pelo menos 27,3\% dos participantes relataram que conheciam a REBRATS (Tabela 4). A variável tipo de segmento e tempo envolvido no controle social não foi estatisticamente significativa. $\mathrm{O}$ Instituto de Avaliação de Tecnologias em Saúde (IATS) foi reconhecido por 20,7\% dos entrevistados e o Boletim Brasileiro de Avaliação de Tecnologias em Saúde (BRATS) foi marcado por $15,3 \%$ e a Colaboração Cochrane foi selecionada por $7,4 \%$ da amostra, com destaque ao segmento de gestores do SUS $(12,1 \% ; \mathrm{p}=0,013)$.

Tabela 4. Fatores associados ao critério "melhoria da qualidade de vida e sobrevida do paciente". Odds ratio (OR) obtido por um modelo de regressão logística com variância robusta, inquérito na $14^{\mathrm{a}} \mathrm{CNS}$.

\begin{tabular}{|c|c|c|c|c|c|c|}
\hline \multirow[t]{2}{*}{ Fatores } & \multicolumn{2}{|r|}{ Todos } & \multicolumn{2}{|c|}{ Usuários $(n=219)$} & \multicolumn{2}{|c|}{$\begin{array}{l}\text { Profissionais de } \\
\text { saúde }(\mathrm{n}=169)\end{array}$} \\
\hline & OR & $\mathrm{p}$-valor & $\mathrm{OR}$ & $\mathrm{p}$-valor & $\mathrm{OR}$ & $\mathrm{p}$-valor \\
\hline $\begin{array}{l}\text { Usa a internet como fonte de } \\
\text { informação sobre tecnologias em } \\
\text { saúde }\end{array}$ & 2,0 & 0,012 & 2,7 & 0,018 & - & NS \\
\hline $\begin{array}{l}\text { Não usa congressos científicos } \\
\text { como fonte de informação sobre } \\
\text { tecnologias em saúde }\end{array}$ & 1,6 & 0,029 & 2,1 & 0,038 & - & NS \\
\hline Prioriza evidências de segurança & 1,9 & 0,004 & 2,0 & 0,029 & - & NS \\
\hline $\begin{array}{l}\text { Prioriza os efeitos observados na } \\
\text { prevenção, diagnóstico, tratamento } \\
\text { ou reabilitação }\end{array}$ & - & NS & - & NS & 2,4 & 0,019 \\
\hline $\begin{array}{l}\text { Prioriza expectativas e } \\
\text { conhecimento dos usuários }\end{array}$ & 2,4 & $<0,001$ & - & NS & 3,0 & 0,028 \\
\hline $\begin{array}{l}\text { Prioriza as implicações para os } \\
\text { serviços de saúde }\end{array}$ & - & NS & - & NS & 2,7 & 0,021 \\
\hline $\begin{array}{l}\text { Prioriza impacto sobre a saúde da } \\
\text { população }\end{array}$ & 1,9 & 0,003 & 2,0 & 0,038 & - & NS \\
\hline $\begin{array}{l}\text { Gostaria de receber informações } \\
\text { sobre avaliação de tecnologias em } \\
\text { saúde }\end{array}$ & 3,8 & 0,013 & 5,6 & 0,004 & - & NS \\
\hline $\begin{array}{l}\text { Não gostaria de participar } \\
\text { do processo de avaliação de } \\
\text { tecnologias em saúde }\end{array}$ & 2,4 & 0,031 & - & NS & - & NS \\
\hline
\end{tabular}

Fonte: questionários respondidos

Nota: NS (não significativo, p>0,05) 
A análise dos fatores associados sobre o conhecimento da REBRATS indicou distintas características dos participantes que não a conheciam (Tabela 5). Para os respondentes que declararam conhecer a REBRATS, houve maior chance de priorizar as expectativas e experiências dos usuários, de saber o que é a ATS, de identificar o IATS e de querer participar de processos na área (Tabela 5).

Tabela 5. Fatores associados ao conhecimento da existência da Rede Brasileira de Avaliação de Tecnologias em Saúde. Odds ratio (OR) obtido por um modelo de regressão logística com variância robusta, inquérito na $14^{\mathrm{a}} \mathrm{CNS}(\mathrm{n}=502)$

\begin{tabular}{|l|l|l|l|}
\hline Fatores & OR & $\begin{array}{l}\text { IC } \\
95 \%\end{array}$ & p-valor \\
\hline $\begin{array}{l}\text { Consulta revistas específicas da área da saúde para obter informações } \\
\text { sobre tecnologias em saúde }\end{array}$ & 1,7 & $1,1-2,8$ & 0,023 \\
\hline $\begin{array}{l}\text { Não consulta a internet para obter informações sobre tecnologias em } \\
\text { saúde }\end{array}$ & 2,1 & $1,1-3,7$ & 0,010 \\
\hline Prioriza expectativas e conhecimentos dos usuários & 1,7 & $1,1-2,8$ & 0,031 \\
\hline $\begin{array}{l}\text { Sabe o que é ou já ouviu falar sobre avaliação de tecnologias em } \\
\text { saúde }\end{array}$ & 4,3 & $2,4-7,8$ & $<0,001$ \\
\hline Conhece o Instituto de Avaliação de Tecnologias em Saúde & 2,0 & $1,2-3,4$ & 0,012 \\
\hline $\begin{array}{l}\text { Gostaria de participar do processo de avaliação de tecnologias em } \\
\text { saúde }\end{array}$ & 2,2 & $1,1-4,6$ & 0,026 \\
\hline
\end{tabular}

Fonte: questionários respondidos

\section{DISCUSSÃO}

O presente estudo apontou que o critério melhoria da qualidade de vida e sobrevida do paciente foi o mais selecionado pela amostra dos participantes da $14^{\text {a }}$ Conferência Nacional de Saúde. Cerca de um terço dos entrevistados relataram conhecer a REBRATS provavelmente em função de pertencerem ao segmento de prestadores e gestores.

O critério mais valorizado foi coerente com as diretrizes definidas na conferência, mas foi possível observar que o tema ATS não compôs o relatório final da conferência, embora houvesse menção a processos relacionados ao tema da inovação e incorporação de novas tecnologias, avaliação de características epidemiológicas para implantação de programas de saúde e atualização de protocolos clínicos (12).

As análises destacam que a expressão qualidade de vida foi citada seis vezes nas quinze diretrizes deliberadas na Conferência (12). Isso significa que existe senso comum sobre a importância desse critério, que coaduna com os achados no inquérito.

Algumas limitações da pesquisa deram ensejo à adoção de cautela na generalização dos resultados. Considerando as circunstâncias de realização do estudo, seria inviável o 
desenvolvimento de uma amostra probabilística, adotando-se, portanto, uma amostra de conveniência realizada em uma população fonte que não pode ser extrapolada. Tal fato não minimizaria a distorção esperada da população acessível no evento, que é familiarizada com o funcionamento e regulação do SUS. Notou-se que a compreensão das perguntas e das opções do questionário foi heterogênea entre os participantes, obrigando os investigadores a disponibilizar técnicos em ATS para o esclarecimento de dúvidas, na tentativa de equalizar o nível de compreensão da pesquisa. Para lidar com essa limitação, investigou-se associações entre as respostas apresentadas, de modo a identificar padrão de preferências na amostra estudada.

Comparando-se com experiências internacionais, esse foi o primeiro estudo realizado em uma conferência de representatividade nacional que identificou os critérios a serem usados para avaliar tecnologias em saúde. Algumas experiências internacionais partiram de inquéritos feitos na internet ou de grupos focais, como pessoas com algum agravo específico, profissionais de saúde ou gestores (13), (14).

Algumas hipóteses podem ser aventadas. $\mathrm{O}$ aumento do acesso à internet influencia o modo de como os usuários do sistema conseguem informações sobre tecnologias em saúde. Por outro lado, pessoas familiarizadas com avaliação de tecnologias em saúde possuem maior chance de procurar periódicos especializados sobre o tema. Podemos supor que os usuários do SUS têm menos chance de participar de congressos científicos sobre saúde ou inovação e incorporação de tecnologias.

O segmento dos usuários que priorizou a melhoria da qualidade de vida e sobrevida do paciente como critério para a adoção e uso de tecnologias para a saúde, como também priorizou evidências de segurança e o impacto sobre a saúde da população. Esse comportamento é semelhante ao encontrado na literatura internacional que discute o envolvimento de pacientes na tomada de decisão sobre ATS (7).

Os profissionais da saúde priorizaram os critérios de impactos nos serviços e os efeitos das tecnologias em saúde, podendo refletir posição frente aos cuidados com os usuários/pacientesPerceber informações sobre avaliação de tecnologias em saúde foi estatisticamente associado ao critério melhoria da qualidade de vida e sobrevida do paciente. Quando todos os entrevistados foram considerados, houve preferência em não participar do processo de avaliação de tecnologias, ocorrendo o inverso quando observados aqueles que declararam conhecer a REBRATS.

Os critérios relacionados a custo para pacientes e/ou sistema de saúde e a relação custo/benefício não foram os mais pontuados. A literatura internacional demonstra que na perspectiva dos gestores de saúde, existe uma preocupação de eficiência dentre os critérios de priorização, referindo-se prioritariamente ao custo-efetividade em vista da efetividade clínica (15) (16).

A escassez de recursos nos sistemas de saúde é melhor percebida pelos gestores e prestadores de serviços. Ao se analisar o relatório final da conferência, há uma diretriz clara para melhoria da gestão e ampliação do financiamento do SUS. Aparentemente, essa convicção não está realacionada com critérios para avaliar e incorporar tecnologias em saúde. 
É provável que a motivação da escolha do critério "melhoria da qualidade e sobrevida do paciente" tenha sido influenciada por uma maior preocupação com o bem-estar individual em relação ao coletivo. O reconhecimento dos valores sociais e a participação popular pode ser uma forma de ajudar a melhorar a qualidade das decisões por trazer informações relevantes, auxiliar na compreensão de outros critérios relevantes e permitir constestação desses públicos (17) (18)(1).

\section{CONCLUSÃO}

Os participantes da $14^{\mathrm{a}}$ Conferência Nacional de Saúde priorizam o critério de valores sociais direcionados à melhoria da qualidade e sobrevida do paciente para avaliar tecnologias em saúde.

Parâmetros relacionados a custos, muito valorizado nos modelos de ATS, tangenciam a preocupação dos segmentos representativos do controle social do SUS. Cerca de um terço dos respondedores conheciam a existência da REBRATS. Aqueles que a identificaram valorizam revistas específicas da saúde e gostariam de participar das avaliações, enquanto o segmento dos usuários prefere receber as informações.

Os achados permitiram ponderar os critérios de avaliação de tecnologias em saúde, como forma de considerar a opinião de usuários e de profissionais envolvidos no controle social do SUS. Ao mesmo tempo, possibilitou identificar a necessidade de estimular a participação social nas consultas à normativa da Lei 12.401, que estabelece requisitos para incorporação de tecnologias. Promover novas estratégias de disseminação da REBRATS, seja por meio de oferta de cursos, seja por meio de mecanismos de tradução do conhecimento para público em geral.

\section{REFERÊNCIAS BIBLIOGRÁFICA}

1. Goodman CS. HTA 101: introduction to health technology assessment [Training Material and Manuals]: U.S. National Library of Medicine; 2009. Available from: http://www.nlm.nih. gov/nichsr/hta101/ta101_c1.html.

2. Silva, H. P.; Petramale, C. A.; Elias, F. T. S. Avanços e desafios da Política Nacional de Gestão de Tecnologias em Saúde. Revista de Saúde Pública (Impresso),

2012, 46: 83-90.. Disponível em: http://www.scielo.br/scielo.php?script=sci arttext\&pid=S0034-89102012000700012 Acesso em 23.abr.2015

3 Donabedian A. The quality of care. How can it be assessed? JAMA. 1988, 260(12):1743-

4. Bastian H, Scheibler F, Knelangen M, Zschorlich B, Nasser M, Waltering A. Choosing health technology assessment and systematic review topics: the development of priority-setting criteria for patients' and consumers' interests. Int J Technol Assess Health Care. 2011; 27(4):348-56. 
5. Health Equality Europe. Understanding health technology assessment. 2013. Disponível em: http://www.htai.org/fileadmin/HTAi_Files/ISG/PatientInvolvement/EffectiveInvolvement/ HEEGuideToHTAforPatientsEnglish.pdf. Acesso em 24.abr.2015

6. Devidas M; STAFINSKI, T. Role of patient and public participation in health technology assessment and coverage decisions. Expert Review of Pharmacoeconomics \& Outcomes Research.2011, 11 (1): 75-89.

7. Health Technology Assessment International. Patient and citizen involvment. 2013. Disponível em: http://www.htai.org/index.php?id=545 Acesso em 24.abr.2015.

8. Littlejohns P, Yeung K, Clark S, Weale A. A proposal for a new social values research program and policy network. J Health Organ Manag. 2012; 26(3):407-21.

9. Banta D, Almeida RT. The development of health technology assessment in Brazil. Int J Technol Assess Health Care. 2009;25 (Suppl 1):255-9.

10. Health Technology Assessment: institutionalization of actions in the Brazilian Ministry of Health. Rev Saúde Pública. 2006;40(4):743-7.

11. Brasil. Lei $n^{\circ} 8.142$, de 28 de dezembro de 1990. Dispõe sobre a participação da comunidade na gestão do Sistema Único de Saúde (SUS \} e sobre as transferências intergovernamentais de recursos financeiros na área da saúde e dá outras providências. Diário Oficial da União $31 \mathrm{dez} 1990$.

12. Brasil. Ministério da Saúde. Conselho Nacional de Saúde. Relatório final da $14^{\text {a }}$ Conferência Nacional de Saúde: todos usam o SUS: SUS na seguridade social: Política pública, patrimônio do povo brasileiro. Brasília: Ministério da Saúde; 2012.

13. Ryan M, Scott DA, Reeves C, Bate A, Van Teijlingen ER, Russell EM, et al. Eliciting public preferences for healthcare: a systematic review of techniques. Health Technol Assess. 2001;5(5):1-186.

14. Varela-Lema L, Merino GA, García ML, Martínez MV, Triana EG, Mota TC. [Requirements for and expectations of health technology assessment in Galicia (Spain). A qualitative study from the perspective of decision-makers and clinicians]. Gac Sanit. 2011;25 (6):454-60.

15. Husereau D, Boucher M, Noorani H. Priority setting for health technology assessment at CADTH. Int J Technol Assess Health Care. 2010; 26(3):341-7.

16. Chalkidou K, Whicher D, Kary W, Tunis S. Comparative effectiveness research priorities: identifying critical gaps in evidence for clinical and health policy decision making. Int J Technol Assess Health Care. 2009 ; 25(3):241-8. 
17. Clark S, Weale A. Social values in health priority setting: a conceptual framework. J Health Organ Manag. 2012; 26(3):293-316.

18. Ahn J, Kim G, Suh HS, Lee SM. Social values and healthcare priority setting in Korea. J Health Organ Manag. 2012; 26(3):343-50.

Artigo apresentado em 08-01-15 Artigo aprovado em 09-09-15 Artigo publicado no sistema em 22-12-15 